Methodology Using Shropshire Doctors Co-operative Ltd (Shropdoc's) recorded data we have collated a representative picture of the palliative care practice over a year period from 161 OOHGP patient interactions.

Results 31\% of home visits (HV) had documentation of potential reversible factors and out of those $72 \%$ were with the patient's estimated prognosis greater than 48 hours. Infection being the most common (57\%) reversible factor, the majority being a lower respiratory tract infection. Overall $5.7 \%$ of $\mathrm{OOH}$ GP palliative HV's resulted in hospital admission, however this decreased to $0.6 \%$ adjusting for an estimate of the patient's prognosis to be less than 48 hours. $16 \%$ were admitted if the patient had a potentially reversible co-existing condition. $33 \%$ of consultations had documentation regarding a continuous subcutaneous infusion (CSCI), $86 \%$ of CSCI interventions were made with a prognosis of less than 48 hours. There were CSCI infusion issues requiring an OOHGP $3.4 \%$ of the time. Overall anticipatory medications were prescribed $39 \%$ of the time.

Conclusion The OOHGP deals with a wide variety of scenarios for a heterogeneous population. The symptoms and treatments instigated are on the most part expected within emergency palliative care. This data begins to quantify and describe the role being performed by OOHGP and has implications for service provision and potentially the necessity of 24 hour specialist palliative provision.

\section{THE SWINBURNE SLOT: A CLINIC-BASED SERVICE FOR DETERIORATING PATIENTS WITH INTERSTITIAL LUNG DISEASE}

${ }^{1}$ Emily Kavanagh, ${ }^{2}$ Eve Palmer, ${ }^{1}$ Julie Harper, ${ }^{2}$ Claire Donaldson, ${ }^{3} \mathrm{~A}$ John Simpson, ${ }^{2}$ Ian Forrest, ${ }^{1,2}$ Anne-Marie Bourke. ${ }^{1}$ Marie Curie Hospice Newcastle; ${ }^{2}$ Newcastle Hospitals NHS Foundation Trust; ${ }^{3}$ Newcastle University

\subsection{6/bmjspcare-2018-ASPabstracts. 140}

Introduction and objectives Patients with interstitial lung disease (ILD) can deteriorate quickly. It can be difficult to determine whether this is due to disease progression or other potentially reversible processes. Such patients are often known to multiple services; carer feedback in 2016 highlighted it is difficult to know who to approach. In response, the Newcastle ILD team introduced a weekly $30 \mathrm{~min}$ rapid access clinic slot (Swinburne Slot, SS) with the aim of determining reversibility and to support patients in their preferred place of care. Our objective was to evaluate this service, which may have practice implications for other teams.

Methods We collected data on how the SS was utilised between 12/09/2016 and 21/03/2017. This included appointment outcome; for example, further imaging or referral to specialist palliative care. Additionally, we collected data on acute hospital admissions within 30 days. Data were obtained using electronic medical records and telephone calls to General Practitioners.

Results The SS was utilised 24 times during the 28 week evaluation period. The most frequent clinic outcomes were adjustment to medication $(n=19)$ and same day palliative care review $(n=12)$. There could be multiple outcomes per patient. Six patients were admitted to hospital within 30 days with an ILD-related problem. Six patients died between the start of the evaluation and the end of data collection.

Conclusions Carer feedback highlighted a deficiency in our service. Our response, the SS, was well utilised, resulting in adjustment to patient management and improved access to palliative care. We present the SS as a workable model which could be replicated by other multidisciplinary teams.

\section{PALLIATIVE CARE AND THE ACUTE STROKE WARD: NEW BEGINNINGS?}

Jeena Ackroyd, Anand Nair. Calderdale and Huddersfield NHS Foundation Trust

10.1136/bmjspcare-2018-ASPabstracts. 141

Background Despite advances in managing stroke, death and severe disability are common outcomes. Many patients unfortunately have profound irreversible damage on a background of multiple co-morbidities. However there is also a group of patients where the extent of irreversibility is unclear and the likelihood of survival may be longer, which leads to complex issues around capacity and decision making, whether clinically assisted nutrition and hydration is appropriate and ongoing symptom management. Difficulties can arise in recognising dying when the trajectory of the disease is not easily predicted.

Method Since May 2016 a member of the palliative care team has attended the board round on the acute stroke ward on a weekly basis to provide direct advice or take referrals while discussing all ward patients.

Results Over 12 months the palliative care team was involved in the care of 37 patients. The average Karnofsky Performance Score (KPS) of patients seen was 20. 57\% referrals were related to managing patients in the last few days of life with $76 \%$ needing advice regarding symptom management. Nearly a third of referrals involved supporting the team in appropriate decision making around escalation of care in particular use of artificial feeding in patients who lack capacity.

Conclusions Stroke patients do have palliative care needs. The acute stroke team valued having regular input from the specialist palliative care team in particular with regard to recognising and managing the last few days of life and help in decision making around long term feeding in patients with an uncertain prognosis who lacked capacity. Funding has been approved to develop this work further with a pilot project enabling a palliative care CNS to have designated time to work on the general stroke wards from 2018.

\section{EVALUATION OF THE USE OF A PATIENT HELD RECORD IN SPECIALIST PALLIATIVE CARE}

Clare White, Jenny Sproule, Paula Brogan, Max Watson. Northern Ireland Hospice, University Ulster

\subsection{6/bmjspcare-2018-ASPabstracts. 142}

Introduction The Royal College of GPs in Northern Ireland created a Patient Held Record (PHR) for patients with progressive, life-limiting illness and their families. This study evaluates its usefulness in the specialist palliative care context.

Methods A prospective longitudinal cohort study through a hospice community service. Patients were given questionnaires with the passport and after 4-6 weeks. Hospice Healthcare Professionals (HCPs) completed a questionnaire and focus group after 8 months.

Results From September 2016-June 2017, 550 patients were screened, 347 (63.1\%) were offered a passport and 259 
accepted it (74.6\% of those offered). 238 patients accepted a research pack, with 105 returning questionnaire 1 (44.1\% response rate), and 26 returning questionnaire 2 (24.8\% of initial respondents). Questionnaire 1 (patients) - 40\% found it difficult or burdensome repeating information to HCPs, and almost 70\% were interested in a PHR to reduce repeating information. Questionnaire 2 (patients)- 74\% found it the passport easy to use, but only a few actually used it regularly. Some felt the passport helped conversations with family and HCPs about day-to-day and future care. Over 60\% stated the PHR was 'very' or 'quite' useful', and would recommend it to others. Questionnaire and focus group with HCPs indicated it was mainly patients and relatives who used the passport, not non-palliative HCPs. Half felt the passport was initially well received, but only $4(22 \%)$ felt it was used regularly by patients or their families. More found it useful to talk about facilitation discussion about future care compared with day-today care.

Conclusions While initial uptake of the passport was high, use appeared low. This evaluation does not support widespread use of a PHR in palliative care, but it may be helpful to individuals, especially earlier in their disease trajectory.

\section{MEASURING THE IMPACT OF END OF LIFE CARE QUALITY IMPROVEMENT (QI) PROJECTS}

S Kite, A Cracknell, A Hurlow, C Iwaniszak, C Pattison, E Rees, A Winfield. The Leeds Teaching Hospitals NHS Trust

\subsection{6/bmjspcare-2018-ASPabstracts. 143}

Aim To demonstrate the application of QI methodology to improving end of life care (EOLC), using improvement data (run charts/statistical process control charts (SPC)) to measure the impact of interventions.

Background QI methods are applied widely across healthcare. SPC and run charts are employed to demonstrate if interventions can lead to sustained and significant improvements. Identifying variables that suit themselves to measurement by repeated data points is more of a challenge for interpersonal aspects of care (such as palliative and EOLC) than for technical interventions (Conry M, 2012).

Methods QI methodology and measures were employed in two QI EOLC initiatives in a large acute NHS trust. The first was a multidisciplinary collaborative to improve the care of patients at risk of clinical deterioration, to reduce avoidable deterioration and/or inappropriate cardiopulmonary resuscitation. The second was an intervention led by the Palliative Care Team to improve the assessment and management of terminal agitation on designated wards. SPC and run charts were created for these initiatives, with baseline data pre-intervention and on-going data collection during the testing, implementation and sustainability phases.

Both initiatives were developed following identification of local need; were led by frontline teams and empowered ward level innovation.

Results In the first initiative SPC charts demonstrated sustained, significant $25 \%$ reductions in cardiac arrest calls across the trust, and on 14 pilot wards a $125 \%$ increase in patients with a treatment escalation plan and $75 \%$ increase in documented CPR decisions.
In the second initiative, run charts demonstrated statistically significant improvements in the rate of assessment, reassessment and evaluation of terminal agitation $(p<0.05)$. Routine review and dissemination of data with the frontline teams in these initiatives enhanced collaborative engagement, motivation and success.

Conclusion SPC and run charts can be used to measure the impact of interventions, and contribute to improvements in EOLC.

\section{UNMET NEEDS FROM MENTAL AND PHYSICAL HEALTH CO-MORBIDITIES: A PROSPECTIVE AUDIT IN COMMUNITY PALLIATIVE CARE}

Sarah Yardley, Monika Gorny. Central and North West London NHS Foundation Trust

10.1136/bmjspcare-2018-ASPabstracts. 144

Background Increasingly patients referred to community specialist palliative care (SPC) have multiple co-morbidities including psychiatric and mental health needs in addition to physical disease. Access to psychiatric services is variable. There is no national standard for community-based shared care in this context and both palliative and psychiatric professionals report challenges.

Methods This prospective audit aimed to:

- establish local need for community psychiatric liaison/shared working;

- identify unmet needs for quality improvement. Patients discussed in a SPC community multidisciplinary team meeting were screened for three months.

Inclusion criteria:

SPC staff had concerns about managing mental health needs; contact with psychiatric services within last year; current medication prescription for psychiatric disorder. Exclusion criteria: not meeting inclusion criteria and dementia if SPC considered this appropriately managed without psychiatric input.

Results In 182 case discussions (May-July 2017), 35 patients (new $n=19 / 96$, complex $n=16 / 86$ ) with unmet mental health needs were identified. 26 had a formal psychiatric diagnosis; 12 had on-going psychiatric care. 20 had never had contact with a local community mental health team, 3 were previously discharged. SPC attempted shared care with mental health services in 10 cases ( 3 via GP, 1 patient refused, 1 via oncology psychology services, 4 received psychiatry services and 1 was accepted but died before being seen). Four patients' needs were first identified by psychiatric liaison teams during hospital admissions. SPC records demonstrate a further 17 patients whose current needs exceeded SPC expertise but no shared care or referrals were attempted. The remaining 4 patients" needs were met directly by SPC. Exemplar vignettes with further learning will be shared.

Conclusions This demonstrates significant unmet mental health needs in our community SPC population. Addressing needs requires proactive liaison between SPC and mental health services with identification of best practice models that also integrate specialist services with general practice. 